\title{
RNA interference-mediated USP22 gene silencing promotes human brain glioma apoptosis and induces cell cycle arrest
}

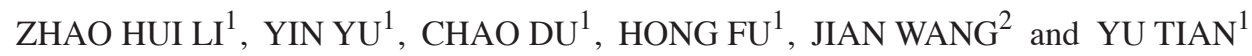 \\ ${ }^{1}$ Department of Neurosurgery, China-Japan Union Hospital of Jilin University, Changchun 130033; \\ ${ }^{2}$ Department of Neurosurgery, Affiliated Hospital of Inner Mongolia University for Nationalities, Tongliao 028000, P.R. China
}

Received October 29, 2012; Accepted February 4, 2013

DOI: $10.3892 / \mathrm{ol} .2013 .1188$

\begin{abstract}
Ubiquitin-specific protease 22 (USP22) is a novel tumor stem cell marker that plays a key role in tumorigenesis and cell cycle progression. However, the effect of silencing the USP22 gene on human brain glioma cell growth is not well understood. In the present study, high gene expression of USP22 was identified in human brain glioma cells. In addition, RNA interference technology was used to silence USP22 gene expression in human brain glioma cells. Silencing the USP22 gene was found to effectively inhibit proliferation of human brain glioma cells, resulting in cell apoptosis and cell cycle arrest at the $G_{2} / M$ phase. USP22 silencing was also found to lead to reduced expression of cell cycle proteins, including CDK1, CDK2 and CyclinB1. In summary, in this study the USP22 gene was demonstrated to play a key regulatory role in the growth of human brain glioma cells by affecting progression of apoptosis and the cell cycle.

\section{Introduction}

Originating in the nerve epithelial tissue, brain glioma is one of the most common malignant tumors in the central nervous system accounting for $>70 \%$ of primary malignant brain tumors (1-3). The high incidence, poor prognosis and low efficacy of therapeutics associated with brain glioma has attracted considerable attention from researchers in the central nervous system disease field; however, progress remains challenging (4). At present, brain glioma is largely treated with surgery, radiotherapy and chemotherapy; however, the curative effect and prognosis are far from optimistic, and there is no significant improvement in treatment efficacy of brain glioma (5-7). In addition, current knowledge on the molecular mechanism of the generation and development of brain glioma remains extremely limited. Analysis of the roles played by
\end{abstract}

Correspondence to: Dr Yu Tian, Department of Neurosurgery, China-Japan Union Hospital of Jilin University, 126 Xiantai Road, Changchun 130033, P.R. China

E-mail: tianyu2801@126.com

Key words: RNA interference, USP22 gene, human brain glioma, cell apoptosis, cell cycle specific molecules in the brain glioma generation and development process is important for the identification of effective treatment approaches and may provide effective molecular targets for future molecular therapies.

A previous study identified an 11-gene Polycomb/cancer stem cell signature that may predict the probability of treatment failure in cancer patients (8). Ubiquitin-specific protease 22 (USP22) is a novel putative cancer stem cell marker involved in the 11-gene Polycomb/cancer stem cell signature (9). USP22 belongs to a large family of proteins with ubiquitin hydrolase activity and has been identified to be an important subunit of the human Spt-Ada-Gen5 acetyltransferase (hSAGA) transcriptional cofactor complex, which is required for activator-driven transcription and cell cycle progression. Within hSAGA, USP22 removes ubiquitin from histone $\mathrm{H} 2 \mathrm{~B}$, thus regulating the transcription of downstream genes associated with epigenetic alteration and cancer progression.

A number of previous studies have reported high expression of USP22 in specific malignant tumors, affecting the progression and prognosis of malignant tumors, including esophageal carcinoma and gastric, colorectal and breast cancer (10-13). To date, the expression of USP22 in human brain glioma cells and its role in cell growth has not been determined. In the present study, high expression of USP22 in human brain glioma cells was identified. RNA interference was used to silence the USP22 gene, leading to apoptosis of human brain glioma cells and induction of cell cycle arrest. In addition, USP22 has been reported to be required for the correct function of MYC, which is widely hypothesized to play a significant role in the regulation of the tumor cell cycle and tumor invasion (14). USP22 has also been demonstrated to inhibit transcription of the p21 gene by deubiquitinating the transcriptional regulator, FBP1, leading to cell proliferation and tumorigenesis (15).

The aim of the present study is to determine the in vitro effect of USP22 gene silencing by RNA interference on human brain glioma cell apoptosis and the cell cycle and to elucidate its molecular mechanism.

\section{Materials and methods}

Cell culture. Human brain glioma U87 and U251 cells were purchased from American Type Culture Collection (Manassas, VA, USA). Cells were cultured in Dulbecco's modified Eagle's medium (DMEM; Gibco-BRL, Carlsbad, CA, USA) 
containing 10\% fetal calf serum (Hyclone Laboratories, Inc., Logan, UT, USA), $100 \mathrm{U} / \mathrm{ml}$ penicillin and $100 \mathrm{U} / \mathrm{ml}$ streptomycin in an incubator containing $5 \% \mathrm{CO}_{2}$ and $95 \% \mathrm{O}_{2}$ at $37^{\circ} \mathrm{C}$. Experimental analyses were performed when cells reached logarithmic growth phase.

The study was approved by the Ethics Committee of the China-Japan Union Hospital of Jilin University, Changchun, China.

Small interfering RNA (siRNA) transfection. Human brain glioma cells were inoculated in 6-well culture plates containing DMEM in the absence of antibiotics at a density of $5 \times 10^{5}$ cell $/ \mathrm{ml}$. Transfection was performed when the cells reached $>60 \%$ confluence. siRNA and Lipofectamine 2000 were added according to the manufacturer's instructions, as described previously (Invitrogen, Carlsbad, CA, USA) (16). The USP22 siRNA sequence was as follows: sense 5'-CACGGACAGUCUCAACAAUTT-3' and anti-sense 5'-AUUGUUGAGACUGUCCGUGTT-3'. Non-specific control siRNA was used as the control group. The efficiency of siRNA interference was determined by RT-PCR and western blot analysis.

$R N A$ extraction and RT-PCR determination. Total RNA was extracted from each experimental group and RNA concentration was calculated according to the manufacturer's instructions (RNAisoTM Plus; Takara Bio, Inc., Shiga, Japan). RT-PCR was performed using a RT-PCR kit obtained from Takara Bio, Inc. and performed in accordance with the manufacturer's instructions. USP 22 and $\beta$-actin primers were synthesized by Invitrogen Life Technologies (Carlsbạd, CA, USA). The primers for amplification were as follows: USP22, 5'-GGACA ACTGGAAGCAGAACC-3' (forward) and 5'-TGAAACAGCCGAAGAAGACA-3' (reverse); $\beta$-actin, 5'-CTGGGACGACATGGAGAAAA-3' (forward) and 5'-AAGGAAGGCTGGAAGAGTGC-3' (reverse). Reaction conditions were as follows: $94^{\circ} \mathrm{C}$ for 2 min followed by 34 cycles of degradation at $94^{\circ} \mathrm{C}$ for $30 \mathrm{sec}$, annealing at $61^{\circ} \mathrm{C}$ for $30 \mathrm{sec}$ and extension at $72^{\circ} \mathrm{C}$ for $30 \mathrm{sec}$. PCR products were subjected to electrophoresis on a $1.0 \%$ agarose gel, then scanned and analyzed with a gel imaging system.

Western blot analysis. Cells of all experimental groups were collected using a cell scraper and $2 \mathrm{ml}$ lysis solution $(50 \mathrm{mM}$ Tris- $\mathrm{HCl}, 137 \mathrm{mM} \mathrm{NaCl}, 10 \%$ glycerin, $100 \mathrm{mM}$ sodium vanadate, $1 \mathrm{mM}$ PMSF, $10 \mathrm{mg} / \mathrm{ml}$ aprotinin, $10 \mathrm{mg} / \mathrm{ml}$ leupeptin, $1 \% \mathrm{NP}-40$ and $5 \mathrm{mM}$ cocktail containing protease inhibitors; pH 7.4) was added to extract proteins. Protein concentation was determined by the BCA method rationing and separated by SDS-PAGE. Next, proteins were transferred to PVDF membranes using the semi-dry method and blocked overnight using 5\% skimmed milk powder at $4^{\circ} \mathrm{C}$. Membranes were washed with TBST and incubated for $1 \mathrm{~h}$ at $37^{\circ} \mathrm{C}$ with primary antibodies against target proteins, followed by an additional TBST wash. Membranes were incubated with appropriate secondary antibodies for $1 \mathrm{~h}$ at $37^{\circ} \mathrm{C}$ and washed with TBST. Antibody reactions were developed by color reaction for $5 \mathrm{~min}$ using autoradiography and Quantity One (Bio-Rad, Hercules, CA, USA) was used to perform optical density analysis and quantification.
Cell viability. Cell viability was determined using the MTT method. Cells $\left(2 \times 10^{4}\right.$ cell/well) were cultured in 96-well culture plates 1 day prior to siRNA transfection. Following adherence to the culture plates, cells were transfected with USP22 and control siRNA on day 2. MTT solution $(5 \mathrm{mg} / \mathrm{ml}$; $20 \mu 1)$ was added to each well and cells were cultured in a $\mathrm{CO}_{2}$ incubator for $4 \mathrm{~h}$. Following this, the culture solution was removed and $150 \mu \mathrm{l}$ DMSO was added to each well and agitated at room temperature for $10 \mathrm{~min}$. OD values of each well were measured using a microplate reader. Analysis for each experimental group was performed in six double wells. Average values were calculated and the growth curves were constructed.

Test of apoptosis with flow cytometry. Cells from all experimental groups were digested in $0.25 \%$ trypsin and resuspended in PBS to prepare single cell suspensions. Cell density was adjusted to $1 \times 10^{6} \mathrm{cell} / \mathrm{ml}$. Next, $5 \mu \mathrm{l}$ Annexin V-FITC and $5 \mathrm{ml}$ PI were added and cells were incubated for $30 \mathrm{~min}$ at $4^{\circ} \mathrm{C}$ prior to analysis by flow eytometry.

Cell cycle analysis, Cells were collected using the trypsin method and washed 3 times with PBS. Following this, cells were fixed at $4{ }^{\circ} \mathrm{C}$ using $75 \%$ cold ethanol overnight. Ethanol was removed and cells were washed 3 times with PBS. Cell density was adjusted to $1 \times 10^{6}$ cells $/ \mathrm{ml}$ at a final volume of $100 \mu \mathrm{l}$ DNAStain comprehensive dye liquor $(500 \mathrm{ml}$; with final concentrations of $50 \mathrm{mg} / \mathrm{l} \mathrm{RNase}, 100 \mathrm{mg} / \mathrm{l} \mathrm{PI}$ and $1 \mathrm{mg} / \mathrm{l}$ Triton X-100) for storage at room temperature in the dark for 15 min prior to analysis by flow cytometry.

Statistical analysis. SPSS 16.0 statistical software was used for statistical analysis. Values are presented as mean $\pm \mathrm{SD}$. Statistical analysis was performed using the Student's t-test. $\mathrm{P}<0.05$ was considered to indicate a statistically significant difference.

\section{Results}

USP22 gene expression in human brain glioma cells. USP22 gene expression was analyzed by RT-PCR. USP22 mRNA was highly expressed in U87 and U251 cells of human brain glioma and USP22 mRNA expression was higher in U87 cells. In addition, western blot analysis of USP22 protein expression identified that protein levels in U87 and U251 cells were consistent with those of USP 22 mRNA. The results indicate that USP22 mRNA and protein are expressed at high levels in U87 and U251 cells of human brain glioma (Fig. 1).

RNA interference-mediated USP22 gene silencing in human brain glioma cells. RNA interference was performed by transfecting U87 and U251 cells with control and USP22 siRNA. Following transfection, RT-PCR and western blot analysis were performed to determine USP22 mRNA and protein expression levels. USP22 siRNA transfection $(58 \mathrm{nM})$ for $24 \mathrm{~h}$ was observed to significantly reduce expression of USP22 mRNA and protein in U87 and U251 cells (Fig. 2A-C). These results indicate that USP22 siRNA transfection for $24 \mathrm{~h}$ effectively silenced USP 22 gene and protein expression. 

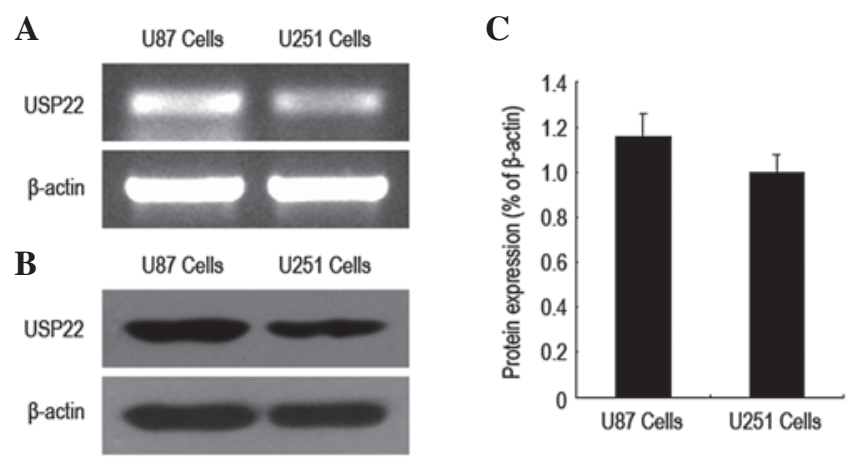

Figure 1. USP22 gene and protein expression in human brain glioma cells. (A) USP22 mRNA expression via RT-PCR analysis. (B) USP22 protein expression via western blot analysis. (C) Histogram of USP22 gene expression levels. Each independent experiment was repeated 3 times. USP22, ubiquitin-specific protease 22 .

A

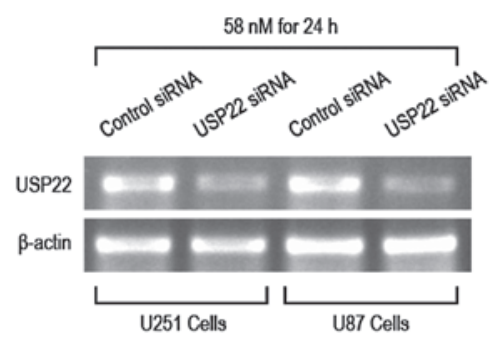

B

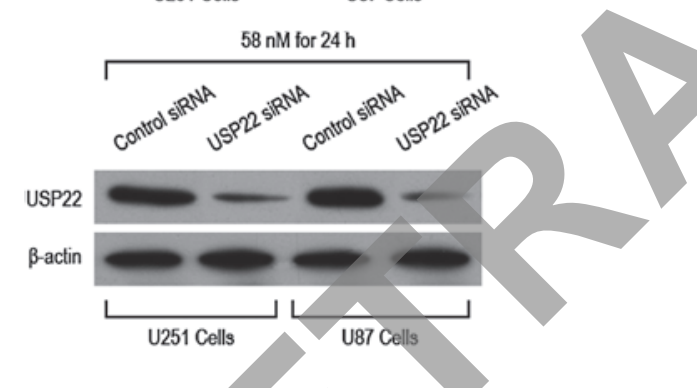

Figure 2. RNA interference to silence USP22 gene expression in human brain glioma cells. U87 and U251 cells were transfected with USP22 siRNA for $24 \mathrm{~h}$ (A) RT-PCR and (B) western blot analysis to determine interference efficiency following transfection. USP22, ubiquitin-specific protease 22.

RNA interference-mediated USP22 gene silencing and growth inhibition of human brain glioma cells. To investigate whether RNA interference-mediated USP22 gene silencing affects the growth of human brain glioma cells, the MTT method was used to analyze the viability of U87 and U251 cells at 12, 24, 48,72 and $96 \mathrm{~h}$ following transfection with USP22 siRNA. The results indicate that the viability of U87 and U251 cells was significantly reduced compared with the control and control siRNA groups. These observations indicate that USP22 gene silencing by RNA interference inhibits growth of human brain glioma cells (Fig. 3A and B).

RNA interference-mediated USP22 gene silencing induced apoptosis of human brain glioma cells. To investigate the molecular mechanism by which USP22 silencing inhibits human brain glioma cell growth, flow cytometry was used to determine the rate of cell apoptosis and western blot analysis was performed to analyze expression levels of apoptosis-related protein changes. Following RNA interference-mediated

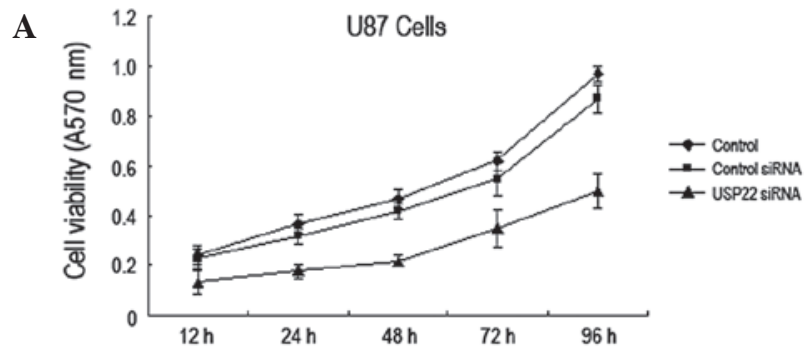

B

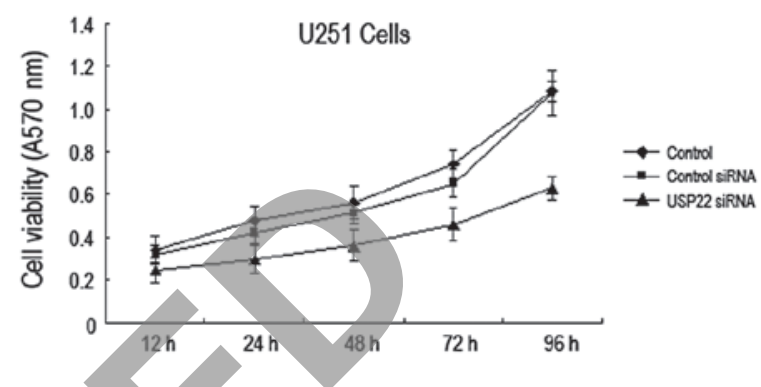

Figure 3. USP22 gene silencing inhibits human brain glioma cell growth MTT was performed to analyze viability of (A) U87 and (B) U251 cells following transfection with USP22 siRNA. Each independent experiment was repeated 3 times. USP22, ubiquitin-specific protease 22.

USP22 gene silencing ( $24 \mathrm{~h})$, the apoptosis rate of U87 and $\mathrm{U} 251$ cells was found to increase significantly (Fig. 4A and B). In addition, protein expression levels of procaspase- $9,-8$ and -3 were markedly reduced (Fig. $4 \mathrm{C}$ and D). The results indicate that inhibition of human brain glioma cell growth by USP22 gene silencing may be relevant to cell apoptosis.

USP22 gene silencing resulted in cell cycle arrest of human brain glioma cells in the $G_{2} / M$ phase. To determine the molecular mechanism by which USP22 gene silencing leads to inhibition of human brain glioma cell growth, the cell cycle was analyzed using flow cytometry. The percentage of USP22 siRNA-transfected U87 and U251 cells entering the $\mathrm{G}_{2} / \mathrm{M}$ phase was significantly higher than that of the control siRNA group (Fig. 5A and B), indicating that the majority of the cells were arrested in the $\mathrm{G}_{2} / \mathrm{M}$ phase. In addition, expression of cell cycle proteins, including CDK1, CDK2, CyclinB1 and CyclinD1, were analyzed by western blot analysis. CDK1, CDK2 and CyclinB1 expression in USP22 siRNA-transfected U87 and U251 cells was markedly reduced. By contrast, the expression of CyclinD1 protein was unchanged (Fig. 6A and $\mathrm{B}$ ). The results indicate that inhibition of human brain glioma cell growth by USP22 gene silencing may induce $\mathrm{G}_{2} / \mathrm{M}$ phase cell cycle arrest via the downregulation of CDK1, CDK2 and CyclinB1 protein expression.

\section{Discussion}

USP22 was recently identified as a novel human deubiquitinating enzyme. High expression levels of USP22 are used to predict the time of recurrence, distant metastasis and treatment failure and correlate with poor prognosis in a number of types of tumor $(11,17,18)$. Previous studies have reported that USP22 silencing inhibits the proliferation of various tumor cells (19-21). However, little is known with regards to the 


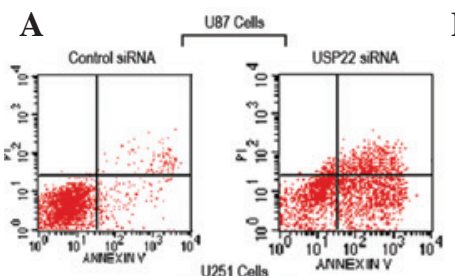

B

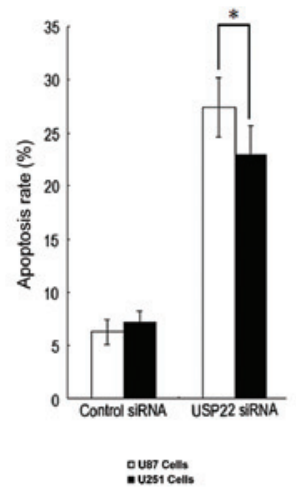

C

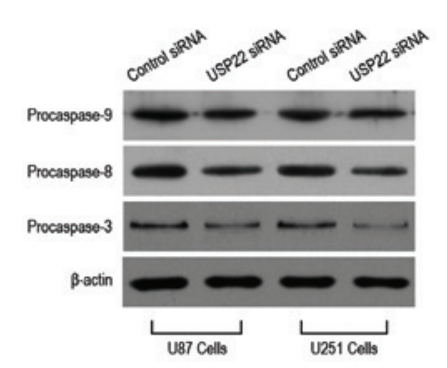

D

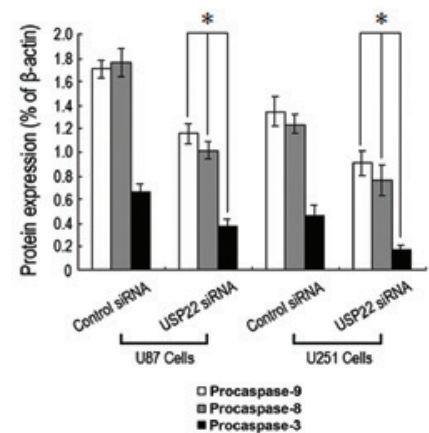

Figure 4. USP22 gene silencing led to human brain glioma cell apoptosis. (A) Flow cytometry to analyze cell apoptosis rate. (B) Quantification of cell apoptosis rate (\%) in U87 and U251 cells. (C) Western blot analysis of procaspase- $-9,-8$ and -3 protein expression. (D) Quantification of procaspase- $9,-8$ and -3 protein expression (\%). Each independent experiment was performed 3 times. $\mathrm{P}<0.05$, vs. control. USP22, ubiquitin-specific protease 22.
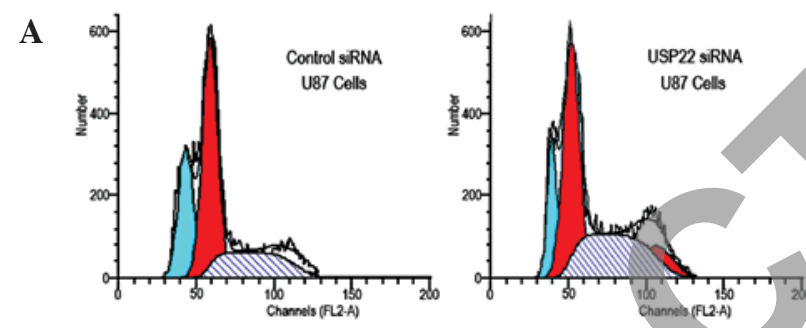

B
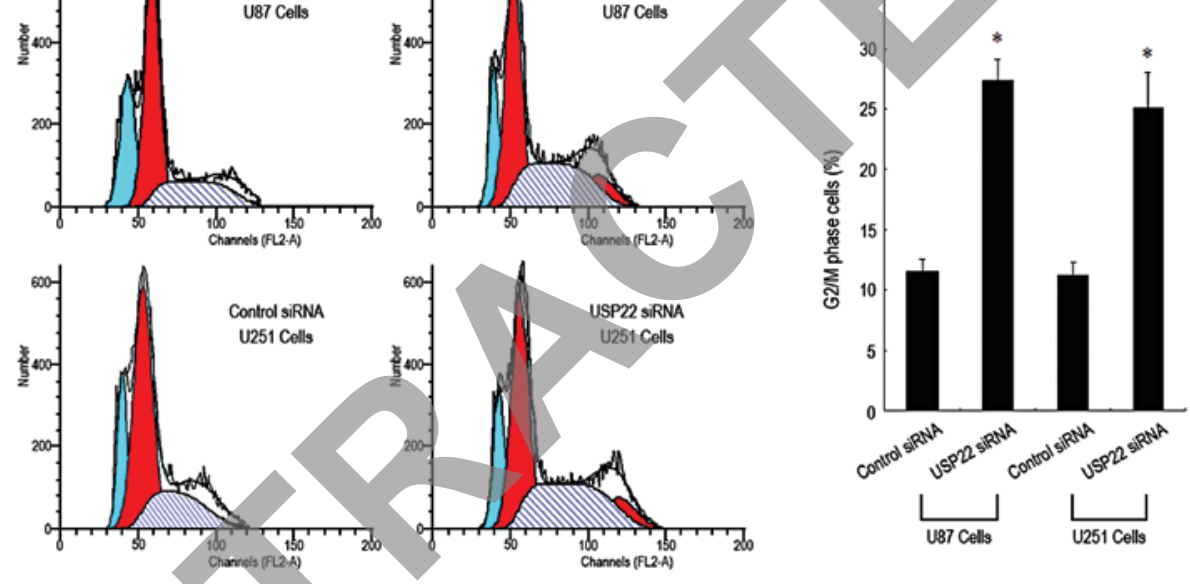

Figure 5. USP22 gene silencing arrested human brain glioma cells in the $\mathrm{G}_{2} / \mathrm{M}$ phase. (A) Flow cytometry to analyze cycle changes in U87 and U251 cells. (B) Quantification of cell cycle changes (\%). Each independent experiment was performed 3 times."P $<0.05$, vs. control. USP22, ubiquitin-specific protease 22 .
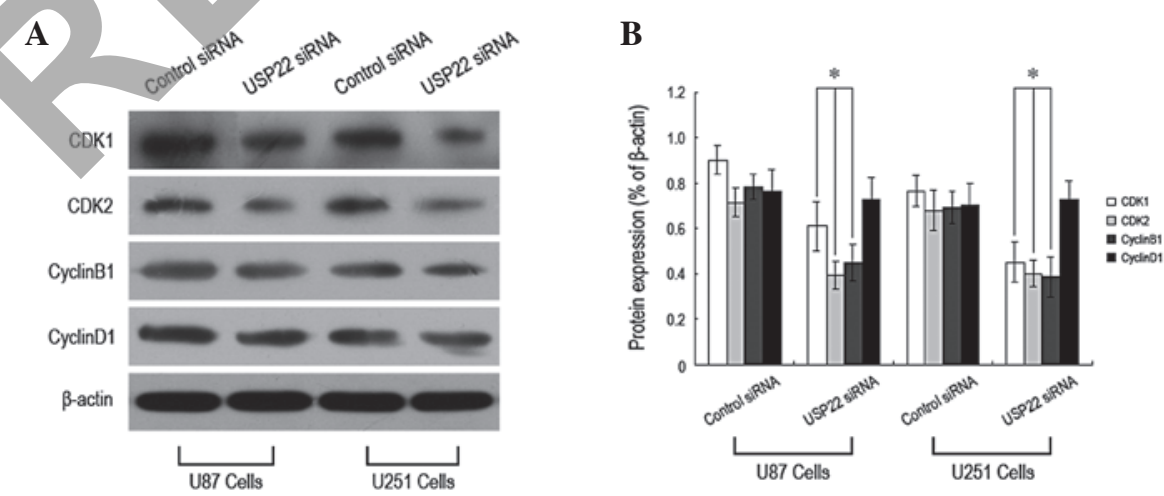

Figure 6. USP22 gene silencing downregulated the expression of CDK1, CDK2 and CyclinB1 protein. (A) Western blot analysis of protein expression of CDK1, CDK2, CyclinB1 and CyclinD1 proteins. (B) Quantification of protein expression of CDK1, CDK2, CyclinB1 and CyclinD1 (\%). "P<0.05, vs. control. Each independent experiment was performed 3 times. USP22, ubiquitin-specific protease 22.

effect of USP22 gene silencing in human brain glioma cells. Therefore, the present study aimed to determine the role of USP22 in human brain glioma and its molecular mechanism. RT-PCR and western blot analysis were performed, revealing high USP22 gene and protein expression in human brain glioma cells, and indicating that USP22 gene expression may represent a novel biological marker and treatment target for brain glioma. 
To establish the effect of the USP22 gene on human brain glioma, small RNA interfering technology was utilized to silence USP22 gene expression in human brain glioma cells. Following this, the biological status of the brain glioma cells was analyzed. Following USP22 gene silencing, the replication rate of brain glioma cells was markedly reduced, indicating that the USP22 gene is involved in the regulation of human brain glioma cell proliferation. Previous studies have demonstrated that the USP22 gene promotes the proliferation of esophageal carcinoma (10) and lung (22) breast (13), colorectal (21) and bladder cancer cells (20). Consistent with these observations, in the present study, USP22 was demonstrated to play an important role in the regulation of human brain glioma cell proliferation.

However, the mechanism by which USPCC gene silencing leads to inhibition of human brain glioma cell proliferation remains unknown. Flow cytometry analysis revealed that human brain glioma cell apoptosis occurred following USP22 gene silencing. Previous studies have reported that USP22 gene silencing induced apoptosis of bladder (20) and colorectal cancer cells (21). Consistent with these results, in the current study, USP22 inhibition of human brain glioma cell proliferation was hypothesized to be markedly associated with apoptosis. In addition, protein expression of procaspase- $9,-8$ and -3 was found to be significantly reduced following USP 22 gene silencing. The activation of members of the caspase family is an important prerequisite for apoptosis (23). The rate of cell apoptosis was also found to increase, indicating that USP22 gene silencing may induce this process in brain glioma cells. Analysis by flow cytometry revealed that USP22 gene silencing arrested human brain glioma cells in the $\mathrm{G}_{2} / \mathrm{M}$ phase. In addition, expression of cell cycle regulatory proteins, including CDK1, CDK2 and CyclinB1, was markedly reduced while no change in the expression of CyclinD1 was identified. These observations indicate that USP22 gene silencing leads to the reduction in the number of human brain glioma undergoing cell division and subsequent inhibition of cell replication.

In the present study, high expression of the USP22 gene in human brain glioma cells was identified for the first time and USP22 gene silencing was found to inhibit human brain glioma cell replication via induction of apoptosis and cell cycle arrest. These observations indicate that the USP22 gene may represent a novel molecular targeted approach for future treatment of brain glioma.

\section{Acknowledgements}

The authors thank Professor Yuzhuo Pan for technical assistance. The present study was supported by grants from the National Science Foundation of China (no. 30672159) and New Century Excellent Talents in Chinese Universities (NCET-06-0306).

\section{References}

1. Ricard D, Idbaih A, Ducray F, Lahutte M, Hoang-Xuan K and Delattre JY: Primary brain tumours in adults. Lancet 379: 1984-1996, 2012.

2. Hess KR, Broglio KR and Bondy ML: Adult glioma incidence trends in the United States, 1977-2000. Cancer 101: 2293-2299, 2004.
3. Johannesen TB, Langmark F and Lote K: Cause of death and long-term survival in patients with neuro-epithelial brain tumours: a population-based study. Eur J Cancer 39: 2355-2363, 2003.

4. Zhang Y, Chao T, Li R, Liu W, Chen Y, Yan X, Gong Y, Yin B, Liu W, Qiang B, Zhao J, Yuan J and Peng X: MicroRNA-128 inhibits glioma cells proliferation by targeting transcription factor E2F3a. J Mol Med (Berl) 87: 43-51, 2009.

5. Komotar RJ, Otten ML, Moise G and Connolly ES Jr: Radiotherapy plus concomitant and adjuvant temozolomide for glioblastoma-a critical review. Clin Med Oncol 2: 421-422, 2008

6. Stummer W and Kamp MA: The importance of surgical resection in malignant glioma. Curr Opin Neurol 22: 645-649, 2009.

7. Stupp R, Mason WP, van den Bent MJ, Weller M, Fisher B, Taphoorn MJ, Belanger K, Brandes AA, Marosi C, Bogdahn U, Curschmann J, Janzer RC, Ludwin SK, Gorlia T, Allgeier A, Lacombe D, Cairncross JG, Eisenhauer E and Mirimanoff RO: Radiotherapy plus concomitant and adjuvant temozolomide for glioblastoma. N Engl J Med 352: 987-996, 2005.

8. Glinsky GV: Death-from-cancer signatures and stem cell contribution to metastatic cancer. Cell Cycle 4: 1171-1175, 2005.

9. Zhang XY, Varthi M, Sykes SM, Phillips C, Warzecha C, Zhu W, Wyce A, Thorne AW, Berger SL and McMahon SB: The putative cancer stem cell marker USP22 is a subunit of the human SAGA complex required for activated transcription and cell-cycle progression. Mol Cell 29: 102-111, 2008.

10. Li J, Wang Z and Li Y: USP22 nuclear expression is significantly associated with progression and unfavorable clinical outcome in human esophageal squamous cell carcinoma. J Cancer Res Clin Oneol 138: 1291-1297, 2012.

11. Yang DD, Cui BB, Sun LY, Zheng HQ, Huang Q, Tong JX and Zhang QF: The co-expression of USP22 and BMI-1 may promote cancer progression and predict therapy failure in gastric carcinoma. Cell Biochem Biophys 61: 703-710, 2011.

12. Liu Y, Yang Y, Xu H and Dong X: Implication of USP22 in the regulation of BMI-1, c-Myc, p16INK4a, p14ARF and cyclin D2 expression in primary colorectal carcinomas. Diagn Mol Pathol 19: 194-200, 2010.

3. Zhang Y, Yao L, Zhang X, Ji H, Wang L, Sun S and Pang D: Elevated expression of USP22 in correlation with poor prognosis in patients with invasive breast cancer. J Cancer Res Clin Oncol 137: 1245-1253, 2011.

14. Zhang XY, Pfeiffer HK, Thorne AW and McMahon SB: USP22, an hSAGA subunit and potential cancer stem cell marker, reverses the polycomb-catalyzed ubiquitylation of histone $\mathrm{H} 2 \mathrm{~A}$. Cell Cycle 7: 1522-1524, 2008.

15. Atanassov BS and Dent SY: USP22 regulates cell proliferation by deubiquitinating the transcriptional regulator FBP1. EMBO Rep 12: 924-930, 2011.

16. Tang B, Du J, Wang J, Tan G, Gao Z, Wang Z and Wang L: Alpinetin suppresses proliferation of human hepatoma cells by the activation of MKK7 and elevates sensitization to cis-diammined dichloridoplatium. Oncol Rep 27: 1090-1096, 2012.

17. Glinsky GV, Berezovska O and Glinskii AB: Microarray analysis identifies a death-from-cancer signature predicting therapy failure in patients with multiple types of cancer. J Clin Invest 115: 1503-1521, 2005.

18. Liu YL, Yang YM, Xu H and Dong XS: Aberrant expression of USP22 is associated with liver metastasis and poor prognosis of colorectal cancer. J Surg Oncol 103: 283-289, 2010.

19. Liu YL, Jiang SX, Yang YM, Xu H, Liu JL and Wang XS: USP22 acts as an oncogene by the activation of BMI-1-mediated INK4a/ARF pathway and Akt pathway. Cell Biochem Biophys 62: 229-235, 2012.

20. Lv L, Xiao XY, Gu ZH, Zeng FQ, Huang LQ and Jiang GS: Silencing USP22 by asymmetric structure of interfering RNA inhibits proliferation and induces cell cycle arrest in bladder cancer cells. Mol Cell Biochem 346: 11-21, 2011.

21. Xu H, Liu YL, Yang YM and Dong XS: Knock-down of ubiquitin-specific protease 22 by micro-RNA interference inhibits colorectal cancer growth. Int J Colorectal Dis 27: 21-30, 2012.

22. Hu J, Liu YL, Piao SL, Yang DD, Yang YM and Cai L: Expression patterns of USP22 and potential targets BMI-1, PTEN, p-AKT in non-small-cell lung cancer. Lung Cancer 77: 593-599, 2012.

23. Nicholson DW, Ali A, Thornberry NA, Vaillancourt JP, Ding CK, Gallant M, Gareau Y, Griffin PR, Labelle M and Lazebnik YA: Identification and inhibition of the ICE/CED-3 protease necessary for mammalian apoptosis. Nature 376: 37-43, 1995. 